Provided for non-commercial research and education use. Not for reproduction, distribution or commercial use.

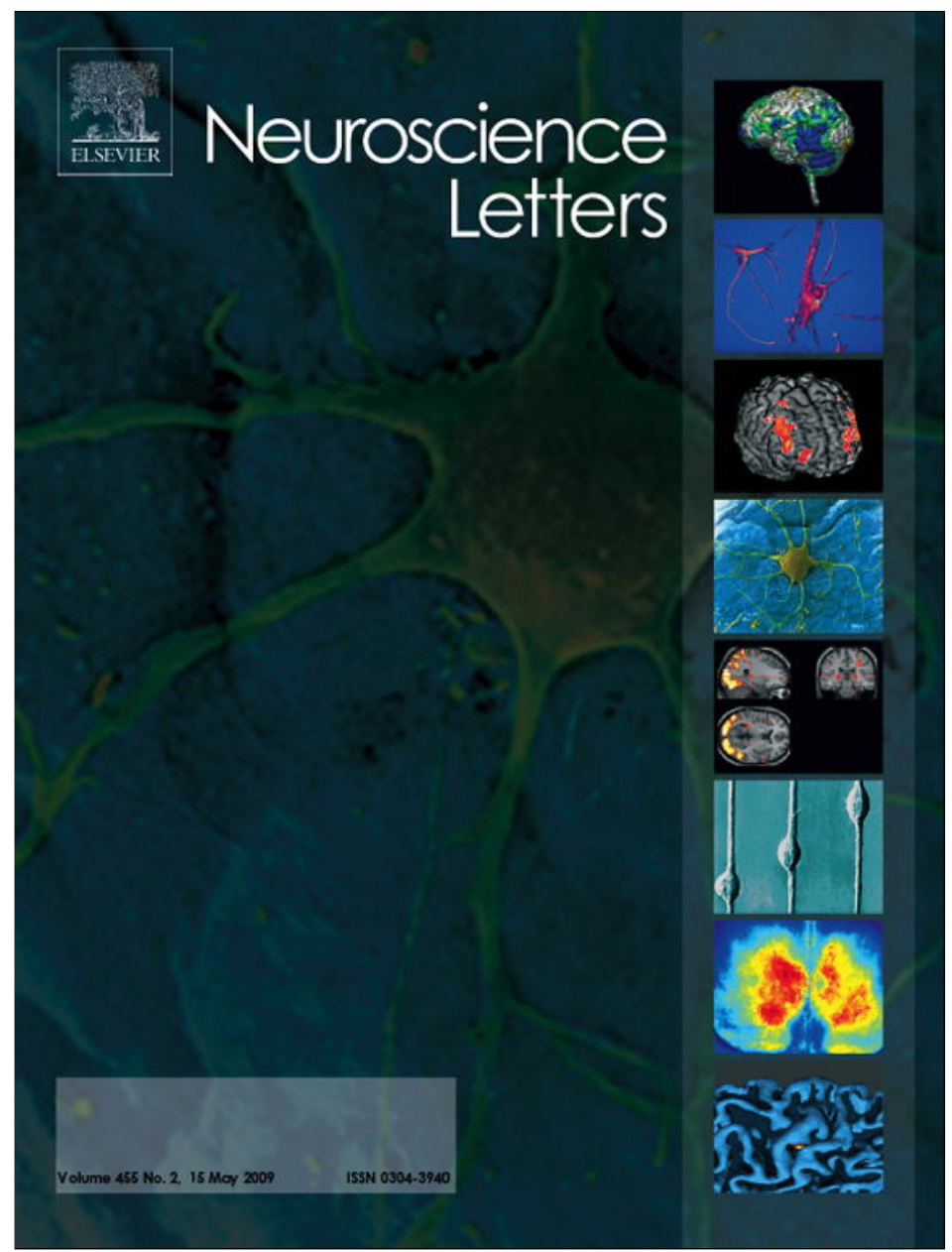

This article appeared in a journal published by Elsevier. The attached copy is furnished to the author for internal non-commercial research and education use, including for instruction at the authors institution and sharing with colleagues.

Other uses, including reproduction and distribution, or selling or licensing copies, or posting to personal, institutional or third party websites are prohibited.

In most cases authors are permitted to post their version of the article (e.g. in Word or Tex form) to their personal website or institutional repository. Authors requiring further information regarding Elsevier's archiving and manuscript policies are encouraged to visit:

http://www.elsevier.com/copyright 


\title{
Crossmodal transfer of emotion by music
}

\author{
Nidhya Logeswaran ${ }^{a}$, Joydeep Bhattacharya ${ }^{\mathrm{a}, \mathrm{b}, *}$ \\ a Department of Psychology, Goldsmiths College, University of London, London SE14 6NW, United Kingdom \\ b Commission for Scientific Visualization, Austrian Academy of Sciences, Vienna A1220, Austria
}

\section{A R T I C L E I N F O}

\section{Article history:}

Received 31 October 2008

Received in revised form 3 March 2009

Accepted 11 March 2009

\section{Keywords:}

Music

Face

Emotion

Priming

ERP

\begin{abstract}
A B S T R A C T
Music is one of the most powerful elicitors of subjective emotion, yet it is not clear whether emotions elicited by music are similar to emotions elicited by visual stimuli. This leads to an open question: can music-elicited emotion be transferred to and/or influence subsequent vision-elicited emotional processing? Here we addressed this question by investigating processing of emotional faces (neutral, happy and sad) primed by short excerpts of musical stimuli (happy and sad). Our behavioural experiment showed a significant effect of musical priming: prior listening to a happy (sad) music enhanced the perceived happiness (sadness) of a face irrespective of facial emotion. Further, this musical priming-induced effect was largest for neutral face. Our electrophysiological experiment showed that such crossmodal priming effects were manifested by event related brain potential components at a very early (within $100 \mathrm{~ms}$ poststimulus) stages of neuronal information processing. Altogether, these results offer new insight into the crossmodal nature of music and its ability to transfer emotion to visual modality.
\end{abstract}

(C) 2009 Elsevier Ireland Ltd. All rights reserved.
Music is often considered as the language of emotion and one of the oldest held views is that music arises principally from human communication-a performer delivers some message to a receptive listener. This message is supposed to be an emotional one and this emotional communication is postulated to be the principal purpose of music [20]. In an extensive review of music performance [9], the analysis of communication accuracy showed that professional music performers are able to communicate basic emotions (e.g., happy, sad, anger) to listeners with an accuracy almost as high as in facial and vocal expression of emotions. Further, there is considerable empirical evidence supporting the statement that emotion is an integral part of a musical experience (see Ref. [10] for a review).

But are musically induced emotions similar to other emotional experiences [23]? An early EEG study [4] demonstrated a characteristic difference in cortical brain activation patterns: positive musical excerpts produced a more pronounced lateralisation towards the left fronto-temporal cortices, whereas negative musical excerpts produced a right fronto-temporal activation pattern. This early result is supported by recent studies showing that left frontal areas are involved with the processing of positive music and right frontal areas with the negative music $[1,8,16]$. Similar frontal asymmetry is well reported for the processing of affective visual stimuli $[2,3]$.

\footnotetext{
* Corresponding author at: Department of Psychology, Goldsmiths College, University of London, New Cross, London SE14 6NW, United Kingdom.

Tel.: +44 2079197334; fax: +44 2079197873.

E-mail address: j.bhattacharya@gold.ac.uk (J. Bhattacharya).
}

Therefore, it is reasonable to infer that there are some overlaps between musical emotions and visual emotions.

But can these musically induced emotions arising through the auditory channel influence our interpretation of emotions arising through other sensory channels (i.e. visual)? Research on crossmodal integration of auditory and visual emotions [5] shows that rating of affective information in one sensory modality can be biased towards the direction of the emotional valence of information in another sensory modality. Event-related-potential (ERP) studies presenting emotionally congruent and incongruent face-voice pairs reveal early ERP effects (N1, P2 components) for congruent face-voice pairs, suggesting an early interaction between auditory and visual emotional stimuli [15,22]. Therefore, musical emotion can interact with visual emotion for simultaneous music and visual processing.

But can musical emotion interact with or even influence the visual emotion for non-simultaneous processes? In other words, can music be used as an affective priming stimulus which could systematically influence the emotional processes of target visual stimuli? Music was earlier used as a priming stimulus in semantic context $[12,21]$. To the best of our knowledge, the current study is the first to address this issue in a crossmodal context by using both behavioural and ERP experiments.

We performed two separate experiments - (i) behavioural and (ii) electrophysiological (EEG) - on a total of 46 adult human participants. Thirty participants ( 15 males and 15 females, mean age $26.1 \pm 4.31$ years) took part in (i) without any cash incentive, and sixteen participants ( 8 males and 8 females, mean age $27.5 \pm 5.88$ years) took part in (ii) against a small cash incentive. All partici- 

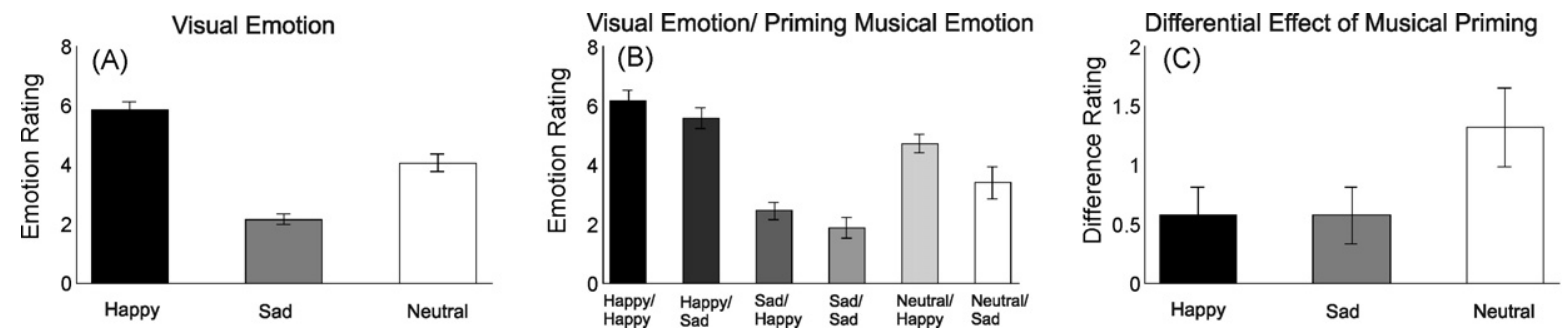

Fig. 1. (a) Emotion ratings of happy, sad and neutral faces, regardless of musical primes. (b) Ratings for six individual conditions: happy, sad and neutral faces primed by happy or sad musical excerpts. (c) Difference (happy - sad) in ratings for three facial emotions. Note that the largest effect was found for neutral facial emotion.

pants were healthy right-handed university students, had normal hearing, normal or corrected-to-normal vision, and had no special musical expertise or musical education. The study was conducted in accordance with the Declaration of Helsinki, and was approved by the Internal Ethics Committee at Goldsmiths College, University of London. All participants gave informed written consent before both experiments.

All musical stimuli were taken from a previous study [1]. Briefly, there were 120 instrumental musical excerpts belonging to two emotional categories: happy and sad. Each piece was played for $15 \mathrm{~s}$ with both beginning and end faded in and out, respectively, to minimize surprise. The visual stimuli were faces of 40 different individuals with each individual showing three types of facial emotions: happy, sad and neutral (http://www.macbrain.org/stim/faces.htm).

There were 90 trials equally divided into six possible conditions ( 2 musical emotions $\times 3$ facial emotions). Each trial lasted for $16 \mathrm{~s}$, where a 15 -s musical excerpt was followed by a facial stimulus presented for $1 \mathrm{~s}$. At the end of each trial, participants were required to rate the facial emotion on a 7 -point scale: $1=$ extremely sad, $2=$ moderately sad, $3=$ slightly sad, $4=$ neutral, $5=$ slightly happy, $6=$ moderately happy, and $7=$ extremely happy. Participants were told to try and to feel the emotion of the musical stimuli and to rely mainly on their feelings while they rated the facial stimuli.

The EEG study followed a similar procedure to the behavioural study but with the following exceptions. There were 120 trials with 20 trials for each condition. Further, instead of an explicit emotional evaluation, the participants were asked to press a button whenever a female face was shown. This minimized the explicit components of emotional processing, and the remaining differences, if any, would reflect the implicitness of emotional processing. Trials were randomized within each block and across participants. EEG signals were recorded from $28 \mathrm{Ag} / \mathrm{AgCl}$ scalp electrodes (Fp1, Fp2, F3, F4, F7, F8, Fz, FC3, FC4, FCz, C5, C6, C3, C4, Cz, CP5, CP6, CP3, $\mathrm{CP} 4, \mathrm{CPz}, \mathrm{P} 7, \mathrm{P} 8, \mathrm{P} 3, \mathrm{P} 4, \mathrm{Pz}, \mathrm{O} 1, \mathrm{O} 2, \mathrm{Oz})$ according to the International 10/20-system. Horizontal and vertical eye movements were recorded from electrodes placed around the eyes. Impedances were kept below $5 \mathrm{k} \Omega$. All scalp electrodes were algebraically referenced to the average of two earlobes. The sampling frequency was $500 \mathrm{~Hz}$.

Perceived emotional ratings were assessed using factorial repeated-measures analysis of variance (ANOVA) with the factors, musical emotion (two levels: happy and sad) and facial emotion (three levels: happy, sad and neutral). Further post hoc comparisons between pairs of specific conditions were carried out by using
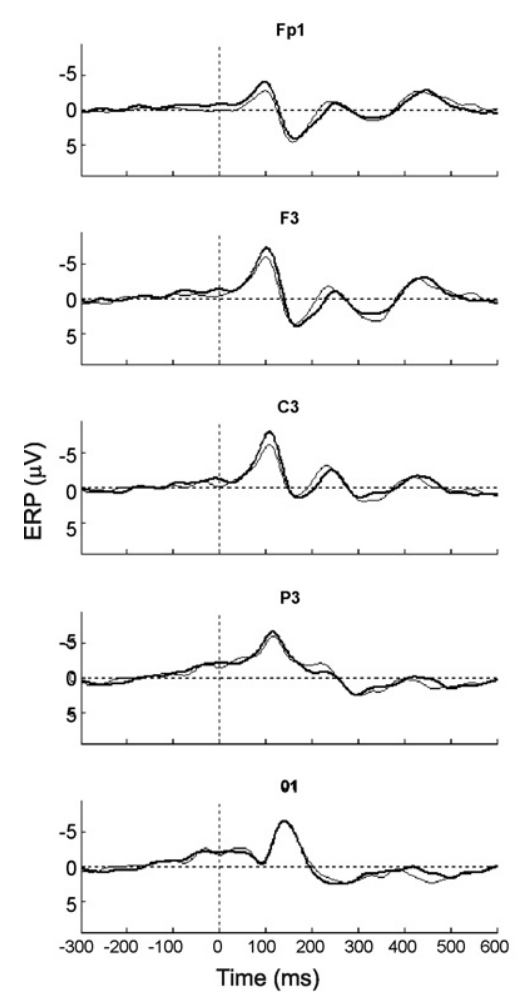
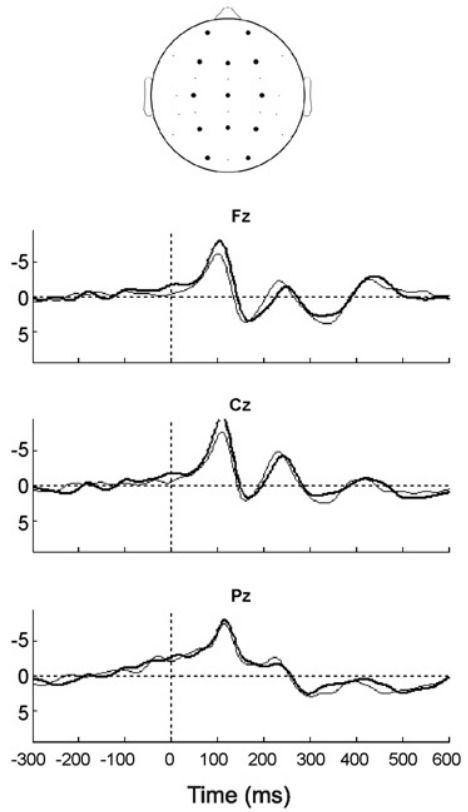

Happy prime Sad prime
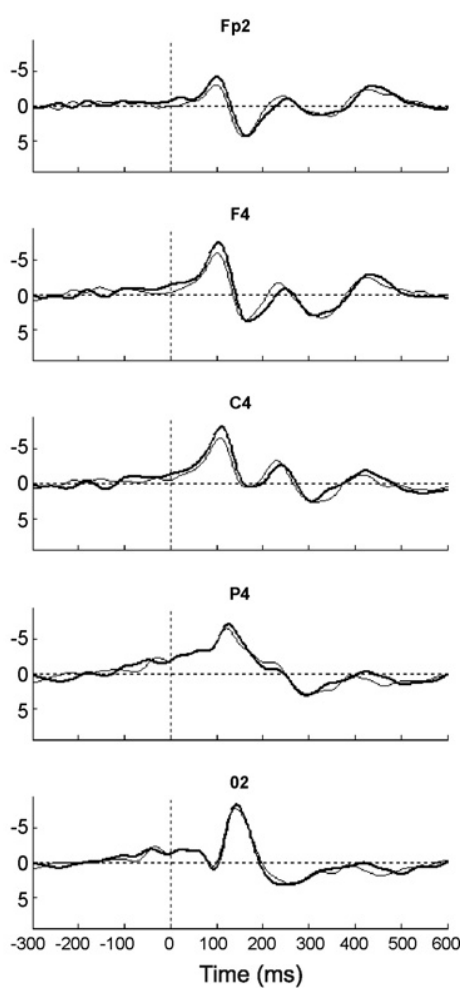

Fig. 2. Grand average ERP profiles at 13 selected electrodes (see the electrode locations on the top) during processing of neutral facial stimuli primed by either happy music (thick line) or sad music (thin line). 
paired $t$-tests. Greenhouse-Geisser correction was applied to adjust for degrees of freedom.

The data pre-processing was carried out by EEGLAB [6]. Stimulus epochs of $1500 \mathrm{~ms}$ starting $500 \mathrm{~ms}$ before the face onset were generated offline from the continuous data. Extracted epochs were subsequently checked for artefacts by visual inspection. In order to correct for ocular artefacts including eye blinks, Independent Component Analysis was performed on the remaining epochs. ERP was obtained by averaging artefact free epochs for each of the six conditions. A series of $2 \times 2$ factorial repeatedmeasures ANOVAs with factors, priming (happy vs. sad) and region (as selected after scalp maps), were conducted on mean ERP amplitudes at specific regions of interest (ROI) between any two conditions with identical facial emotion but with different musical primes. Temporal regions of interest were selected on the basis of global field power (GFP) [13] which quantifies the instantaneous global activity across the spatial potential fields. Spatial regions of interest were selected on the basis of scalp maps of mean ERP amplitudes at the pre-selected temporal region of interest. Across statistical comparison, we found that spatial regions of interest consisted of two levels-anterior and posterior. Instead of a datablind procedure of selecting region of interests on an ad-hoc basis, this data-driven method selected only a few regions of interests, thereby minimizing the error variance and maximizing the effect size.

Fig. 1(a) shows the mean ratings of happy, neutral and sad faces regardless of the type of musical primes. It was clear that the facial emotions were rightly rated and classified by our participants. Mean ratings for six conditions, separately, are shown in Fig. 1(b). The happy faces when primed by happy music were rated $(6.13 \pm 0.36)$ higher (i.e. more happy) than when primed by sad music $(5.56 \pm 0.35)$, and the sad faces when primed by sad music were rated $(1.87 \pm 0.30)$ lower (more sad) than when primed by happy music $(2.44 \pm 0.30)$. Further, when the neutral faces were primed by happy music, the rating $(4.68 \pm 0.33)$ was much higher than when it was primed by sad music $(3.37 \pm 0.54)$. Therefore, the differential effects of priming (happy-sad) were similar for happy and sad faces (mean difference rating of 0.57 ) but was almost doubled (mean difference rating of 1.31) for neutral face (Fig. 1(c)). Repeated-measures ANOVA showed that there were highly significant main effects for musical emotion $(F(1,29)=103.29, p<0.001)$ and facial emotion $(F(2,28)=1358.89$, $p<0.001)$. The music $\times$ faces interaction effect was also found to be highly significant $(F(2,58)=34.37, p<0.001)$. These results show that the effect of musical priming was largest for emotionally neutral target stimuli.

ERPs were always compared between two conditions with same facial emotion but with different priming, and same analysis procedure was applied for all three types of facial emotions. Since the behaviour study showed largest effect for neutral faces, we strategically emphasized the results for neutral faces in details as follows, and the results for other two types of facial emotions will be briefly mentioned.

Visual inspections revealed that ERP profiles for neutral faces primed by happy music were markedly different from those primed by sad music (Fig. 2). Enhanced N1 component was seen across all frontal and central electrodes for happy, as opposed to sad, musical primes. The classical N170 face component was exhibited in occipital and parietal (P7, P8, not shown) regions bilaterally for both priming conditions. Between 180 and $250 \mathrm{~ms}$, an increased positivity (or reduced negativity) for happy primes as compared to sad primes was noticed in frontal, central. At a later stage $(300 \mathrm{~ms}$ onward), posterior positivity was observed for both conditions. GFP values were plotted in Fig. 3 (top panel) and the two profiles were separated as early as from $50 \mathrm{~ms}$ till $150 \mathrm{~ms}$, and then again for the time period $190-210 \mathrm{~ms}$. Scalp maps at these time windows were
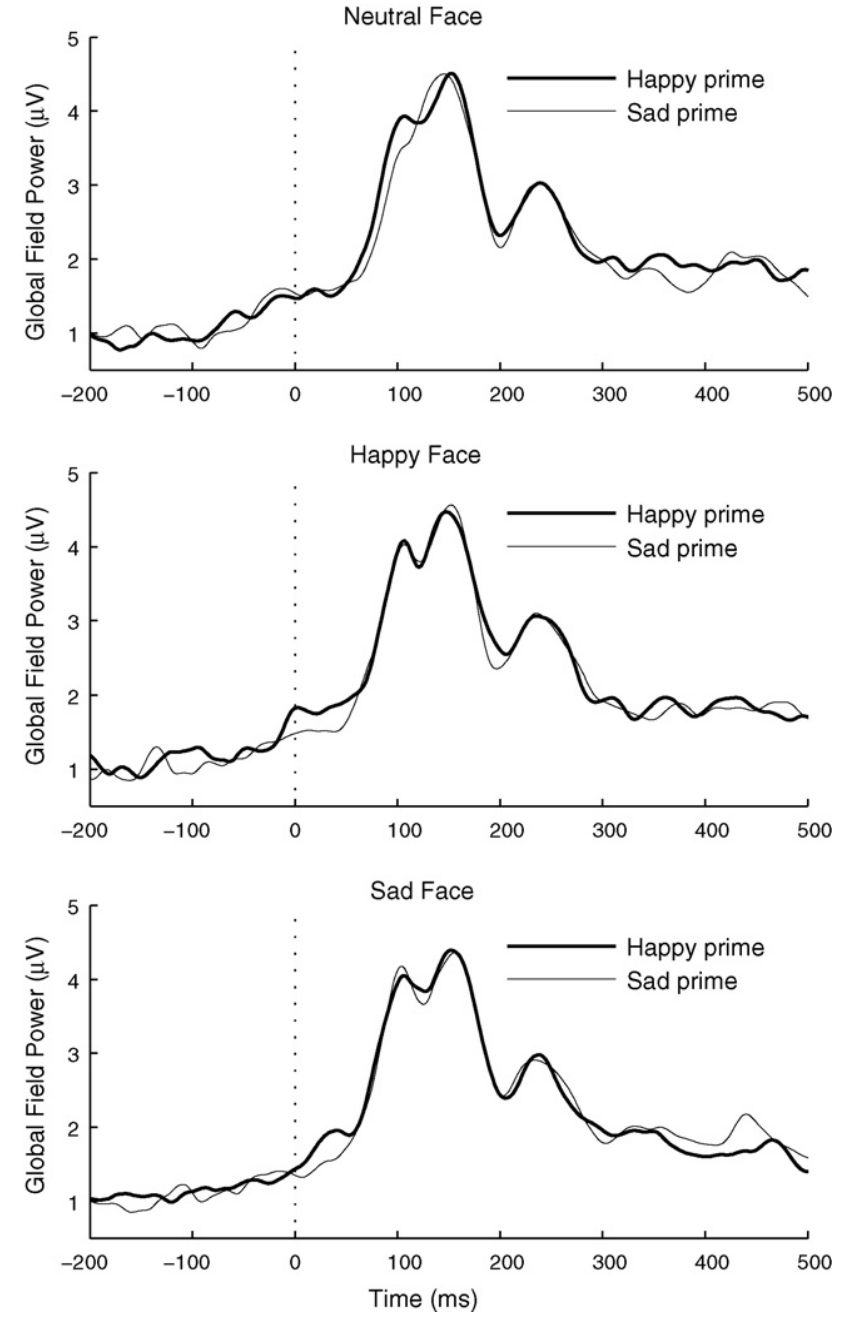

Fig. 3. Global field power values for three emotional facial stimuli: neutral face (upper panel), happy face (middle panel), and sad face (lower panel). For each emotion type, two types of musical priming, happy and sad, were shown in thick and in thin lines, respectively. The high GFP values correspond to pronounced potential fields with high peaks (both positive and negative) and steep gradients, whereas low GFP values correspond to flat potential fields. For each facial emotion, time windows where the two profiles showed maximal separation between two musical priming were used for successive statistical analysis.

displayed in Fig. 4 for both conditions and their differences. As compared to negative musical prime, positive musical prime showed an enhanced negativity in frontal and central brain regions during 50-150 ms and enhanced positivity during $190-210 \mathrm{~ms}$ in similar anterior brain regions. For the $\mathrm{N} 1$ time window ( $50-150 \mathrm{~ms})$, statistical analysis showed a significant effect of priming $(F(1,15)=5.35$, $p=0.03)$, and a priming $\times$ region interaction effect $(F(1,15)=8.62$, $p=0.01)$. For the later time window $(190-210 \mathrm{~ms})$, a significant priming effect $(F(1,15)=4.66, p=0.047)$ was found.

GFP plots for other two types of facial emotions were shown in Fig. 3 (middle and lower panels). For happy facial stimuli, differences between happy and sad musical primes were found between 0-50 and $160-210 \mathrm{~ms}$. Statistical analysis of mean ERP amplitudes showed a near significant priming effect during $160-210 \mathrm{~ms}$ $(F(1,15)=4.33, p=0.06)$ and an almost significant priming $\times$ region interaction effect between 160 and $210 \mathrm{~ms}(F(1,15)=4.16, p=0.06)$. For sad facial stimuli, the early $(0-50 \mathrm{~ms})$ difference between the two priming conditions was also found, but the later differences were found during $430-450 \mathrm{~ms}$. Statistical analysis of mean ERP amplitudes showed significant region effects, $F(1,15)=7.54$, $p=0.015$ and $F(1,15)=32.29, p<0.001$, for both time windows, 

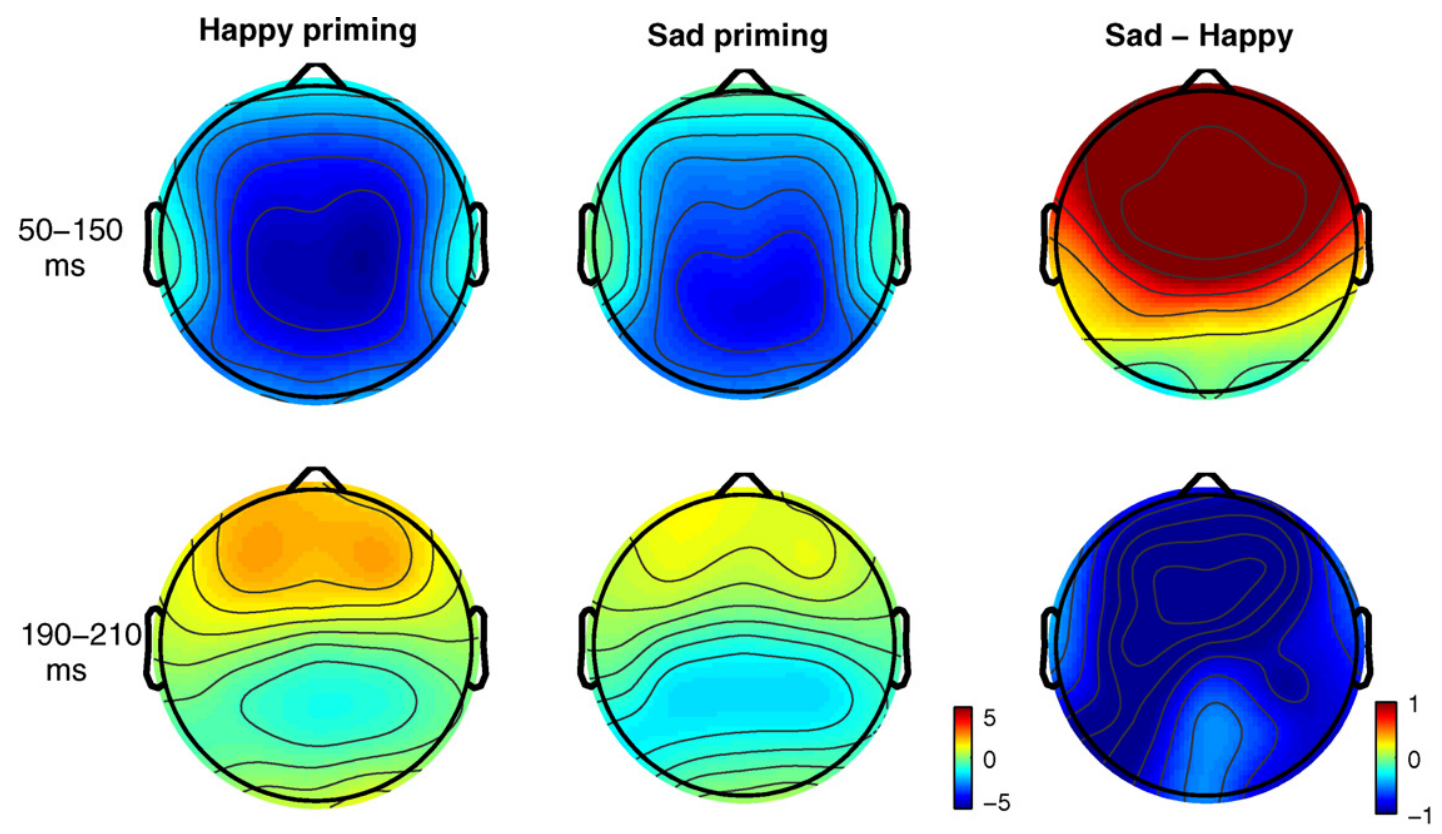

Fig. 4. The scalp distribution of mean ERP amplitudes at two different time windows (50-150 and 190-210 ms) for target neutral faces primed by happy (left column) and sad (middle column) musical stimuli. The right column shows the same but for the difference potentials (sad - happy). As compared to sad musical prime, happy musical prime produced enhanced negativity at the first time window and enhanced positivity at the second time window, both over anterior brain regions.

$0-50 \mathrm{~ms}$ and $430-450 \mathrm{~ms}$, respectively, and a near significant effect of priming $(F(1,15)=4.30, p=0.06)$ was found at the later time window.

Our behavioural experiment confirmed that emotional rating of the target facial stimuli could be biased towards the direction of the emotional valence of the musical primes. Earlier research $[5,22]$ reported that emotions in auditory stimuli interact with emotions in visual stimuli for simultaneously presented auditory-visual stimuli. But our result extends it further by showing that such interaction could also occur for non-simultaneous processing, i.e. when the emotional auditory stimuli precede the emotional visual stimuli. In other words, priming musical stimuli can systematically influence the perceived emotional contents in target visual stimuli. Music was earlier used in semantic priming paradigms. Using chord sequences, either consonant or dissonant, as priming stimuli, it was shown [21] that target words are faster recognized for emotionally congruent chord-word pairs than for incongruent ones. Further, when target words are preceded by semantically unrelated musical primes, N400, an ERP component reflecting contextual integration, effect is reported [12]. Altogether, this suggests that music has an excellent potential to be used as an emotional priming stimulus.

Our behaviour data also shows that the largest effect of musical priming was found for neutral faces with an effect size almost twice those for happy or sad faces. It was shown earlier [14] that as compared to emotionally distinct (i.e. happy, angry, sad) facial stimuli, emotionally ambiguous (i.e. neutral) facial stimuli are more likely to be influenced by auditory cues in a facial emotion detection task. The information-content of neutral faces are supposedly lower than those of happy or sad faces, and since the brain relies on cues from multiple sensory stimuli to create an optimal representation of the external environment, emotionally neutral stimuli is being influenced by emotionally conspicuous stimuli, even though being generated by different senses. Although in our paradigm, there is no such explicit requirement of integration of information across musical and visual stimuli, our findings suggest that a generic mechanism of multimodal affective interaction might exist also in a priming paradigm. Alternatively, this could also be explained by the affect-as-information hypothesis [19] which relies on the assumption that affective processes mainly occur outside of awareness. In contrast to traditional assumption of judgement and decision making which emphasizes the role of target related features, this hypothesis states that when making evaluative judgements, participant often ask themselves, "How do I feel about it?" [18], and therefore, "they may mistake feelings due to a pre-existing state as a reaction to the target" [17]. Since the emotionally neutral facial stimuli contain less information than emotionally conspicuous (happy or sad) facial stimuli, the transient affect from the priming musical stimuli has the maximal impact in determining the evaluation of the neutral facial stimuli.

Our ERP data showed that for neutral facial emotion, happy music, as compared to sad music, showed a significant effect during the $\mathrm{N} 1$ time period $(50-150 \mathrm{~ms})$. Earlier Pourtois et al. [15] have found that simultaneous presentation of emotionally congruent face-voice pairs produce an enhancement of auditory $\mathrm{N} 1$ component as compared to incongruent pairs, suggesting an early crossmodal binding. In contrast to this study which reported enhancement over auditory cortex, our N1 effect was predominant over fronto-central and midfrontal regions (FC3/4, FCz, Fz, Cz). Taken together, this suggests that happy or positive auditory emotion is more likely to influence neutral visual emotion by engaging brain regions responsible for top-down projections.

ERP results also showed priming related enhancement of P2 (190-210 ms) component for happy and neutral target faces but not for sad target faces. Similar modulation of P2 has also recently been reported [22] for happy picture-voice pairs presented simultaneously but not for sad pairs. Enhanced positivity at similar time window has also been found for processing isolated emotional faces as compared to neutral faces [7]. However, the functional role of P2 is not yet clear (but see Ref. [22] for some possible explanations) in mediating interaction between priming musical stimuli and emotionally selective (happy and neutral, but not sad) target faces.

Finally, let us offer a few practical remarks. First, the current paradigm of music-induced emotional priming is quite different from other mood-induction procedures which are associated with a longer lasting change of emotional states, whereas our study investigated the effect of emotional changes on a much shorter time scale [11]. Secondly, unlike previous ERP studies of facial emotion 
processing, we always compared the same facial emotional type but differed only in priming. Therefore, our results indicate a more subtle component in early neural responses which can potentially bias subsequent emotional evaluation occurring at later stages. This was also manifested by our robust behavioural findings which called for an explicit evaluation of facial emotions. Thirdly, our ERP data primarily reflects an implicit emotional processing since the participants were naïve with respect to the specific aims of the study. Further, as the task of gender detection did not require the participants to focus on the presented emotions, the results are less likely to be attributed to differences in directed attention as a function of presented emotions. Therefore, the ERP results suggest an early processing of emotional facial expression primed by musical stimuli.

In summary, the results of our behavioural and ERP study revealed some patterns of crossmodal influences by musical emotion. Behavioural data clearly showed that listening to musical excerpts, albeit short, could significantly influence the subsequent explicit evaluation of visual emotional stimuli. ERP data showed that such musical priming could also influence implicit visual emotional processes.

\section{Acknowledgements}

The study was supported by JST.ERATO Shimojo project (JB). We are thankful to Prof. Eckart Altenmüller for the music stimuli, to Rob Davis for technical support, to Job Lindsen for help in data pre-processing, and to Prof. Rolf Reber for his helpful comments as a reviewer. Author contributions: J.B. conceived research; N.L. collected data; J.B. and N.L. analyzed data and wrote the paper.

\section{References}

[1] E. Altenmuller, K. Schurmann, V.K. Lim, D. Parlitz, Hits to the left, flops to the right: different emotions during listening to music are reflected in cortical lateralisation patterns, Neuropsychologia 40 (2002) 2242-2256.

[2] T. Canli, J.E. Desmond, Z. Zhao, G. Glover, J.D.E. Gabrieli, Hemispheric asymmetry for emotional stimuli detected with fMRI, Neuroreport 9 (1998) 3233-3239.

[3] R.J. Davidson, Anterior cerebral asymmetry and the nature of emotion, Brain and Cognition 20 (1992) 125-151.

[4] R.J. Davidson, G.E. Schwartz, C. Saron, J. Bennett, D.J. Goleman, Frontal versus parietal EEG asymmetry during positive and negative affect, Psychophysiology 16 (1979) 202-203.
[5] B. de Gelder, J. Vroomen, The perception of emotions by ear and by eye, Cognition \& Emotion 14 (2000) 289-311.

[6] A. Delorme, S. Makeig, EEGLAB: an open source toolbox for analysis of singletrial EEG dynamics including independent component analysis, Journal of Neuroscience Methods 134 (2004) 9-21.

[7] M. Eimer, A. Holmes, Event-related brain potential correlates of emotional face processing, Neuropsychologia 45 (2007) 15-31.

[8] E.O. Flores-Gutierrez, J.L. Diaz, F.A. Barrios, R. Favila-Humara, M.A. Guevara, Y. del Rio-Portilla, M. Corsi-Cabrera, Metabolic and electric brain patterns during pleasant and unpleasant emotions induced by music masterpieces, International Journal of Psychophysiology 65 (2007) 69-84.

[9] P.N. Juslin, P. Laukka, Communication of emotions in vocal expression and music performance: different channels, same code? Psychological Bulletin 129(2003) $770-814$.

[10] P.N. Juslin, J.A. Sloboda (Eds.), Music and Emotion, Oxford University Press, Oxford, 2001, p. 487.

[11] P.N. Juslin, D. Vastfjall, Emotional responses to music: the need to consider underlying mechanisms, The Behavioral and Brain Sciences 31 (2008) 559-575 (discussion 575-621).

[12] S. Koelsch, E. Kasper, D. Sammler, K. Schulze, T. Gunter, A.D. Friederici, Music, language and meaning: brain signatures of semantic processing, Nature Neuroscience 7 (2004) 302-307.

[13] D. Lehmann, W. Skrandies, Reference-free identification of components of checkerboard-evoked multichannel potential fields, Electroencephalography and Clinical Neurophysiology 48 (1980) 609-621.

[14] D.W. Massaro, P.B. Egan, Perceiving affect from the voice and the face, Psychonomic Bulletin \& Review 3 (1996) 215-221.

[15] G. Pourtois, B. de Gelder, J. Vroomen, B. Rossion, M. Crommelinck, The timecourse of intermodal binding between seeing and hearing affective information, Neuroreport 11 (2000) 1329-1333.

[16] D.L. Santesso, L.A. Schmidt, L.J. Trainor, Frontal brain electrical activity (EEG) and heart rate in response to affective infant-directed (ID) speech in 9-month-old infants, Brain and Cognition 65 (2007) 14-21.

[17] N. Schwarz, Feelings as information: informational and motivational functions of affective states, in: E.T. Higgins, R. Sorrentino (Eds.), Handbook of Motivation and Cognition: Foundations of Social Behaviour, vol. 2, Guildford Press, New York, 1990, pp. 527-561.

[18] N. Schwarz, G.L. Clore, How do I feel about it? The informative function of mood in: K. Fiedler, J. Forgas (Eds.), Affect, Cognition and Social Behaviour, C.J. Hogrefe, Toronto, 1988, pp. 44-62.

[19] N. Schwarz, G.L. Clore, Mood, misattribution, and judgments of wellbeing-informative and directive functions of affective states, Journal of Personality and Social Psychology 45 (1983) 513-523.

[20] M. Serafine, Music as Cognition: The Development of Thought in Sound, Columbia University Press, New York, 1988.

[21] B. Sollberger, R. Reber, D. Eckstein, Musical chords as affective priming context in a word-evaluation task, Music Perception 20 (2003) 263-282.

[22] K.N. Spreckelmeyer, M. Kutas, T.P. Urbach, E. Altenmuller, T.F. Munte, Combined perception of emotion in pictures and musical sounds, Brain Research 1070 (2006) 160-170

[23] L.J. Trainor, L.A. Schmidt, Processing emotions induced by music, in: I. Peretz, R. Zatorre (Eds.), The Cognitive Neuroscience of Music, Oxford University of Press, Oxford, 2007, pp. 310-324. 